\title{
Recovery characteristics of total intravenous anesthesia with propofol versus sevoflurane anesthesia: a prospective randomized clinical trial
}

This article was published in the following Dove Press journal: Journal of Pain Research

\author{
Ozlem Kocaturk' \\ Sultan Keles ${ }^{2}$ \\ 'Department of Oral and Maxillofacial \\ Surgery, Division of Anesthesiology, \\ Faculty of Dentistry, Adnan Menderes \\ University, Aydin, Turkey; ${ }^{2}$ Department \\ of Pediatric Dentistry, Faculty of \\ Dentistry, Adnan Menderes University, \\ Aydin, Turkey
}

Background: Pediatric dental procedures are performed under anesthesia because children may be uncooperative in the dental clinic due to their young age. Emergence delirium (ED), which involves a variety of behavioral disturbances that are frequently observed in children following emergence from general anesthesia, remains an unclear phenomenon. The aim of this randomized controlled trial is to compare the incidence of ED in children who underwent full mouth dental rehabilitation under either sevoflurane (SEVO) anesthesia or propofol-based total intravenous anesthesia (TIVA).

Patients and methods: One hundred and twenty children with American Society of Anesthesiologists status I-II, aged $\geq 3$ and $\leq 6$ years, undergoing dental rehabilitation were assigned to receive either TIVA or SEVO. ED and postoperative pain were evaluated by a blinded investigator using the Pediatric Anesthesia Emergence Delirium scale and the Face, Legs, Activity, Cry, Consolability scale every $5 \mathrm{~min}$. The recovery time, satisfaction levels of parents or guardians, extubation time, duration of the operation, and type of dental procedure were also recorded.

Results: Data of 116 subjects were analyzed. The incidence of ED was higher after SEVO than after TIVA ( 65.5 vs $3.4 \%, P=0.00)$. Greater postoperative pain was observed in the SEVO group (median 3 vs $1, P=0.000$ ). A statistically significant, moderate correlation ( $\mathrm{rs}=0.46, P<0.0001$ ) was found between the Face, Legs, Activity, Cry, Consolability and Pediatric Anesthesia Emergence Delirium scores. A higher parental satisfaction level was observed in the TIVA group. Conclusion: A lower incidence of ED and a higher parental satisfaction level were observed after TIVA. Moreover, TIVA resulted in a more comfortable postoperative period due to reduced postoperative pain, and the extubation time and recovery time were not increased.

Keywords: emergence delirium, sevoflurane, propofol, TIVA, child, dental surgery

\section{Introduction}

Rapid awakening after the use of insoluble anesthetics has been proposed to initiate emergence delirium (ED) by worsening a child's underlying sense of apprehension when finding him/her in an unfamiliar environment. ${ }^{1}$ Sikich and Lerman ${ }^{2}$ defined ED as "a disturbance in a child's awareness of and attention to his/her environment with disorientation and perceptual alterations including hypersensitivity to stimuli and hyperactive motor behavior in the immediate post-anesthesia period." The incidence of ED largely depends on age, anesthetic technique, surgical procedure, and administration of adjunct medication. The incidence of ED in pediatric anesthesia, based on the use of inhaled agents, may be as high as $40 \%$ and is highest in children aged 2-6 years. ${ }^{1}$
Correspondence: Ozlem Kocaturk Department of Oral and Maxillofacial Surgery, Division of Anesthesiology, Faculty of Dentistry, Adnan Menderes University, Hasan Efendi St, Aydin 09100, Turkey

Tel +902562133939

$\mathrm{Fax}+902562151918$

Email ozlemoget2002@yahoo.com 
The ideal anesthetic should allow fast emergence and a short recovery without postoperative side effects as well as a rapid and smooth induction for pediatric outpatient anesthesia. $^{3}$

Sevoflurane (SEVO) exhibits low blood and tissue solubility, resulting in rapid elimination for outpatient anesthesia. ${ }^{4,5}$ Recent reports suggest that SEVO may be associated with a higher incidence of postoperative ED. ${ }^{6}$

Propofol is widely used in outpatient anesthesia because of its ideal anesthetic characteristics of providing a smooth and fast recovery with few postoperative side effects. ${ }^{7}$ Chandler et $\mathrm{al}^{8}$ showed a lower incidence of ED after total intravenous anesthesia (TIVA) than that after SEVO anesthesia in children aged 2-6 years following strabismus surgery. Additionally, they stated that TIVA with propofol (TIVA-p) provided more favorable results in terms of recovery characteristics, including rescue medications, ED, and recovery time.

Indications for the use of general anesthetics for comprehensive dental treatment are an inability to cooperate because of extreme fearfulness or anxiety, lack of emotional maturity, and mental, physical, or medical disability. ${ }^{9}$ A limited number of studies are available comparing SEVO with TIVA-p in terms of recovery characteristics among children undergoing comprehensive dental treatment.

We designed this randomized clinical trial to compare the ED and recovery characteristics after SEVO anesthesia and TIVA-p in children undergoing outpatient dental treatment.

\section{Patients and methods}

This prospective, randomized, controlled clinical trial was approved by the Research Ethics Committee of the Adnan Menderes University Faculty of Dentistry (ref no 2017/17). This trial was also registered (Protocol Registration Receipt NCT03330613) at http://www.clinicaltrials.gov. Written informed consent was obtained from the parents or guardians of all study participants.

In accordance with the Declaration of Helsinki, a total of 116 children with American Society of Anesthesiologists status I or II, aged 3-7 years, undergoing comprehensive dental treatment under general anesthesia were recruited between November 2017 and January 2018. The exclusion criteria included pharyngeal pathology, known pulmonary or cardiovascular disease, developmental delay, neurologic and psychiatric diagnoses, abnormal lipid or carbohydrate metabolism, $<3$ rd or $>97$ th percentile weight for age, mental retardation, previous anesthetic complications, contraindication to either anesthetic regimens, and lack of parental consent for the child's participation in the study.

After obtaining written informed consent, all patients were randomly assigned to one of the two groups, with 58 patients in each. The patients were assigned to a group based on a code within a sealed, coded, opaque envelope chosen by a ward nurse on the day of the operation, and the randomization process was supervised by a single author. The subjects were randomized to receive induction and maintenance of anesthesia with either SEVO or TIVA-p.

\section{SEVO anesthesia group}

All patients in the SEVO group were intubated by the same anesthetist $(\mathrm{OK})$. In addition to standard monitoring, the patients' bispectral index (BIS; Covidien Inc., Boulder, CO, USA) values were recorded. Mask induction was performed with mixture of $60 \% \mathrm{~N}_{2} \mathrm{O}$ in $40 \% \mathrm{O}_{2}$ for $60 \mathrm{~s}$ followed by incremental increases in inspired SEVO (1\%-7\%). Following loss of consciousness, an intravenous line was established, and then $0.5 \mathrm{mg} / \mathrm{kg}$ Myocron $^{\circledR}$ (rocuronium; Vem, Istanbul, Turkey), $1 \mu \mathrm{g} / \mathrm{kg}$ of Ultiva ${ }^{\circledR}$ (remifentanil; Glaxo Wellcome, Marly-le-Roi, France), and a bolus of $1 \mathrm{mg} / \mathrm{kg}$ of lidocaine were administered, followed by nasotracheal intubation with a cuffed tube. Anesthesia was maintained with a mixture of $60 \% \mathrm{~N}_{2} \mathrm{O}$ in $40 \% \mathrm{O}_{2}$ and a titration of SEVO $(1 \%-4 \%)$. Supplementary doses of $0.5 \mu \mathrm{g} / \mathrm{kg}$ of remifentanil were administered when BIS value $>60$ until the end of the surgery.

\section{TIVA-p group}

After establishing an intravenous line, anesthesia was induced with $1.5 \mu \mathrm{g} / \mathrm{kg}$ of remifentanil and $2.5 \mathrm{mg} / \mathrm{kg}$ of propofol and then a bolus of $1 \mathrm{mg} / \mathrm{kg}$ of lidocaine and $0.5 \mathrm{mg} / \mathrm{kg}$ of rocuronium were administered, followed by nasotracheal intubation with a cuffed tube. Anesthesia was maintained with an infusion of propofol at $6-12 \mathrm{mg} / \mathrm{kg} / \mathrm{h}$ and remifentanyl was titrated at $0.5-1 \mu \mathrm{g} / \mathrm{kg} / \mathrm{min}$ by the anesthesiologist. During this procedure, anesthetic depth was evaluated via BIS value.

\section{Dental treatment procedures}

All procedures were performed by the same pediatric dentist (SK). Decayed teeth underwent either restorative (compomer resin, composite) or endodontic (pulpotomy, pulpectomy) procedures. Teeth that could not be restored were extracted. The number of dental procedures, the duration of the dental operation, and the duration of anesthesia were noted. Postoperative pain control was provided with paracetamol $2.5 \mathrm{mg} / \mathrm{kg}$ intravenously for all patients. 


\section{Postanesthesia outcome measures}

Once all the procedures were completed, the patients were extubated and transferred to the postanesthesia care unit (PACU). A research nurse, who was blinded to the anesthesia method, recorded the data regarding postoperative outcomes. The following outcome parameters were recorded: postoperative pain, ED, recovery time, satisfaction levels of parents or guardians, and extubation time. The extubation time was calculated as the time from the end of anesthesia to extubation of the nasotracheal tube.

In the PACU, the patients were evaluated at 5 min intervals using the Aldrete scale. The Aldrete scoring system is used to clinically evaluate the physical status of patients recovering from general anesthesia. A score of $0-2$ is given for each of the five categories (activity, circulation, consciousness, $\mathrm{O}_{2}$ saturation, and respiration) with a maximum score of 10. Recovery time was calculated from the patient's arrival to the PACU to the achievement of an Aldrete score of $\geq 9$.

The primary endpoint of the study was ED. The Pediatric Anesthesia Emergence Delirium (PAED) scale is used to assess patients on the following five psychometric items:

1. the child makes eye contact with the caregiver;

2. the child's actions are purposeful;

3. the child is aware of his or her surroundings;

4. the child is restless; and

5. the child is inconsolable.

Items $1-3$ are reversed scored as follows: $4=$ not at all; $3=$ just a little; $2=$ quite a bit; $1=$ very much; and $0=$ extremely. Items 4 and 5 are scored as follows: $0=$ not at all; $1=$ just a little; $2=$ quite a bit; $3=$ very much; and $4=$ extremely. The scores of each item are summed to obtain a total PAED score. ${ }^{2}$ ED increases directly with the total score. A PAED score of $\geq 10$ signified the presence of ED.

Postoperative pain intensity was assessed using the Face, Legs, Activity, Cry, Consolability (FLACC) scale. Responses in each category are scored between 0 and 2 , for a maximum total score of 10. A pain score is obtained by reviewing the descriptions of behavior in each of the FLACC categories and selecting the number that most closely matches the observed behavior. The numbers obtained for each category are added to obtain the total pain score, which will be between 0 and $10 .{ }^{10}$ Observational scoring was performed immediately after the patient was sufficiently alert to make purposeful movements. Children were observed by the same blinded nurse for a total of $35 \mathrm{~min}$ in the PACU.

The PAED and FLACC scores were evaluated every $5 \mathrm{~min}$ during the observation period, and the highest scores were recorded for analysis. The parents or guardians were asked to grade their satisfaction levels with their children's recovery from 0 to $10(0=$ very dissatisfied; $10=$ very satisfied $)$.

\section{Statistical analyses}

The sample size (58 patients per group) was determined using the GPower software, based on a study by Chandler et $\mathrm{al},{ }^{8}$ to detect a reduction of $>50 \%$ (from $38.3 \%$ to $14.9 \%$ ) in ED with a power of $80 \%$ using a Student's $t$-test to compare means with an alpha value of 0.05 .

The SPSS program for Windows (Version 20.0; IBM Corporation, Armonk, NY, USA) was used for the statistical analysis. All data were subjected to the Kolmogorov-Smirnov test for normality. Two sample $t$-tests were used to compare age, weight, duration of the operation, duration of anesthesia, number of dental procedure types, recovery time, extubation time, and guardians' satisfaction levels between the two groups. Chi-square tests were used to compare the presence of ED between the two groups. The relationship between PAED and FLACC scores was investigated using Spearman's rank correlation coefficient. A 5\% type 1 error level was used to determine statistical significance.

\section{Results}

One hundred twenty patients were recruited, and 116 patients were enrolled in this study. Figure 1 shows the patient recruitment and follow-up processes. No significant differences were observed in age, gender, weight, number of dental procedure types, duration of the operation, and anesthesia duration between the two groups $(P>0.05)$ (Table 1).

In the PACU, a statistically significant difference in the incidence of ED was observed between the groups. A total of $65.5 \%$ of the subjects in the SEVO group had PAED scores $\geq 10$ and $3.4 \%$ of the subjects in the TIVA-p group had PAED scores $\geq 10(P=0.00)$ (Figure 2).

A statistically significant difference was observed between the groups in FLACC scores, with a higher median value in the SEVO group than in the TIVA-p group (Table 2). Children who experienced ED had a higher maximum FLACC score (median=4; IQR=2-5) than children who were not affected by ED (median=2; IQR=1-3) $(P<0.05)$ (Figure 3$)$. A statistically significant, moderate correlation $(\mathrm{rs}=0.46, P<0.0001)$ was found between FLACC and PAED scores.

No statistically significant differences were found between the groups for extubation and recovery times $(P>0.05)$.

No statistically significant differences were found between the groups for heart rate, systolic arterial pressure, diastolic 


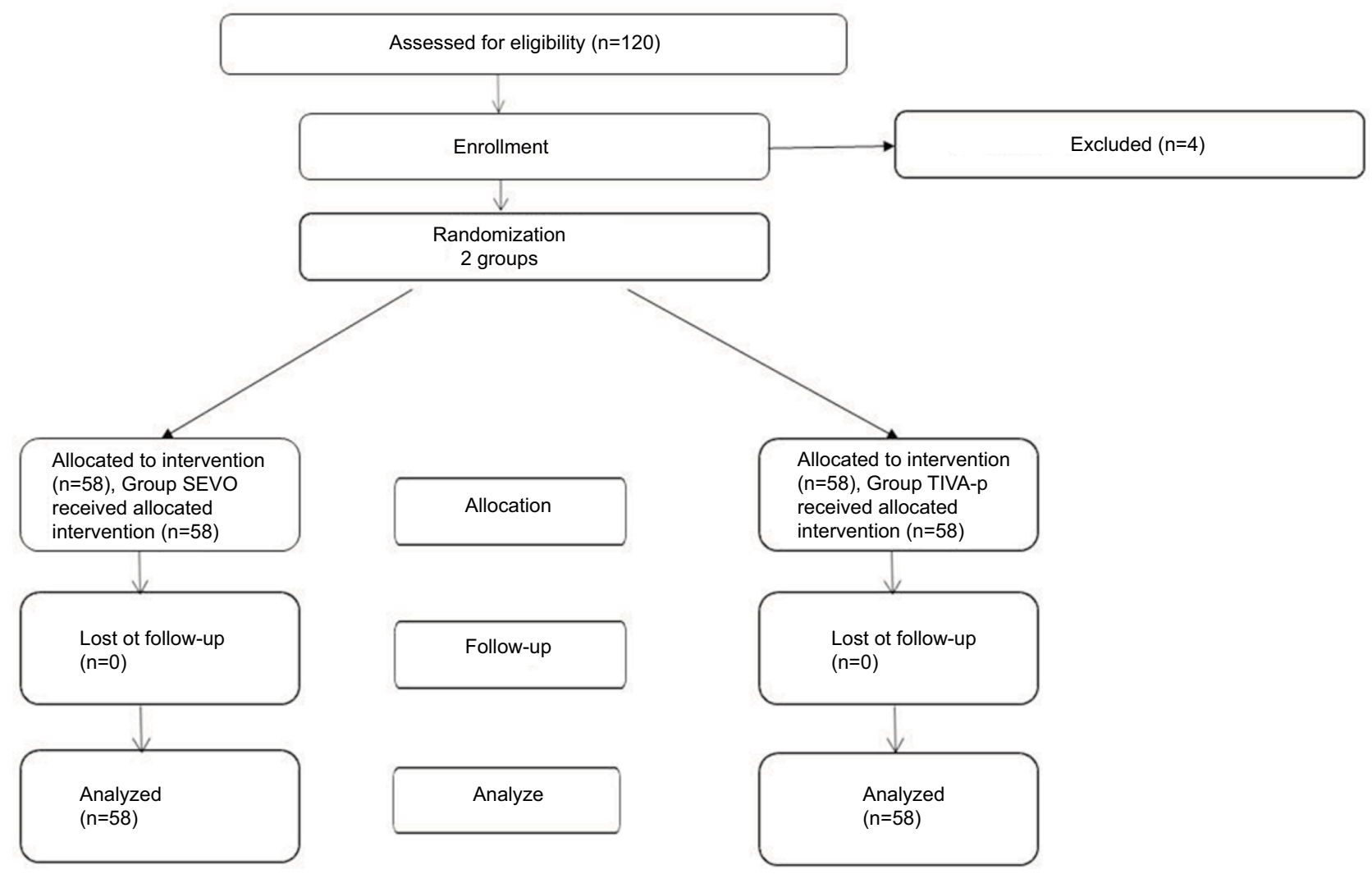

Figure I Flowchart of the study.

Abbreviations: SEVO, sevoflurane; TIVA-p, total intravenous anesthesia with propofol.

Table I Patient demographics and perioperative characteristics of patients

\begin{tabular}{llll}
\hline Patient characteristics & SEVO & TIVA-p & P-value \\
\hline Age (years) & $4.89 \pm 1.32$ & $4.67 \pm 1.39$ & 0.37 \\
Weight (kg) & $18.01 \pm 3.57$ & $18.0 \pm 3.55$ & 0.97 \\
Gender (male/female) & $31 / 27$ & $28 / 30$ & 0.71 \\
ASA classification (I/II) & $43 / 15$ & $46 / 12$ & 0.66 \\
$\begin{array}{l}\text { Procedure, n (\%) } \\
\text { Restorative }\end{array}$ & $18(31.1)$ & $17(29.3)$ & 0.84 \\
$\begin{array}{l}\text { Restorative and extraction } \\
\text { and/or endodontic }\end{array}$ & $40(68.9)$ & $41(70.7)$ & \\
$\begin{array}{l}\text { Duration of anesthesia (min) } \\
\text { Duration of operation (min) }\end{array}$ & $91.6 \pm 24.1$ & $88.6 \pm 23.7$ & 0.49 \\
\hline
\end{tabular}

Note: Data are presented as mean \pm SD.

Abbreviations: ASA, American Society of Anesthesiologists; SEVO, sevoflurane; TIVA-p, total intravenous anesthesia with propofol.

arterial pressure, peripheral oxygen saturation levels at PACU arrival and at discharge from the PACU $(P>0.05)$. The global satisfaction scores of parents were significantly higher in the TIVA-p group than in the SEVO group $(P<0.05)$ (Table 2$)$.

\section{Discussion}

This randomized controlled clinical trial revealed a lower incidence of ED and lower pain scores (FLACC) after TIVA-p than after SEVO anesthesia in children aged between 3 and
7 years following comprehensive dental treatment. Additionally, a higher parental satisfaction level was observed in the TIVA-p group. Both groups were comparable in terms of extubation time and recovery time.

Many previous studies have found that the incidence of ED after SEVO anesthesia was higher than that after TIVA-p, which is similar to our results. ${ }^{11,12}$ The incidence of ED was reportedly between $40 \%$ and $80 \%$ depending on various factors, such as rapid awakening in an unfamiliar environment, immaturity, and variable recovery, resulting in a dissociative state and pain sensation. Cravero et al ${ }^{11}$ found a significantly greater incidence (80\%) of emergence agitation (EA) in children anesthetized with SEVO for MRI scans. Nakayama et al $^{12}$ reported an incidence of $42 \%$ for EA after SEVO anesthesia and an incidence of only $5 \%$ after propofol anesthesia in preschool-aged children. We found an ED incidence of $65.5 \%$ in the SEVO group versus $3.4 \%$ in the TIVA-p group. The differences between the incidences of ED observed in these studies may be due to the type of surgery, duration of the operations, age range, and differences in the evaluation scales used.

The PAED scale proposed by Sikich and Lerman ${ }^{2}$ is a reliable and valid tool that may minimize measurement errors 


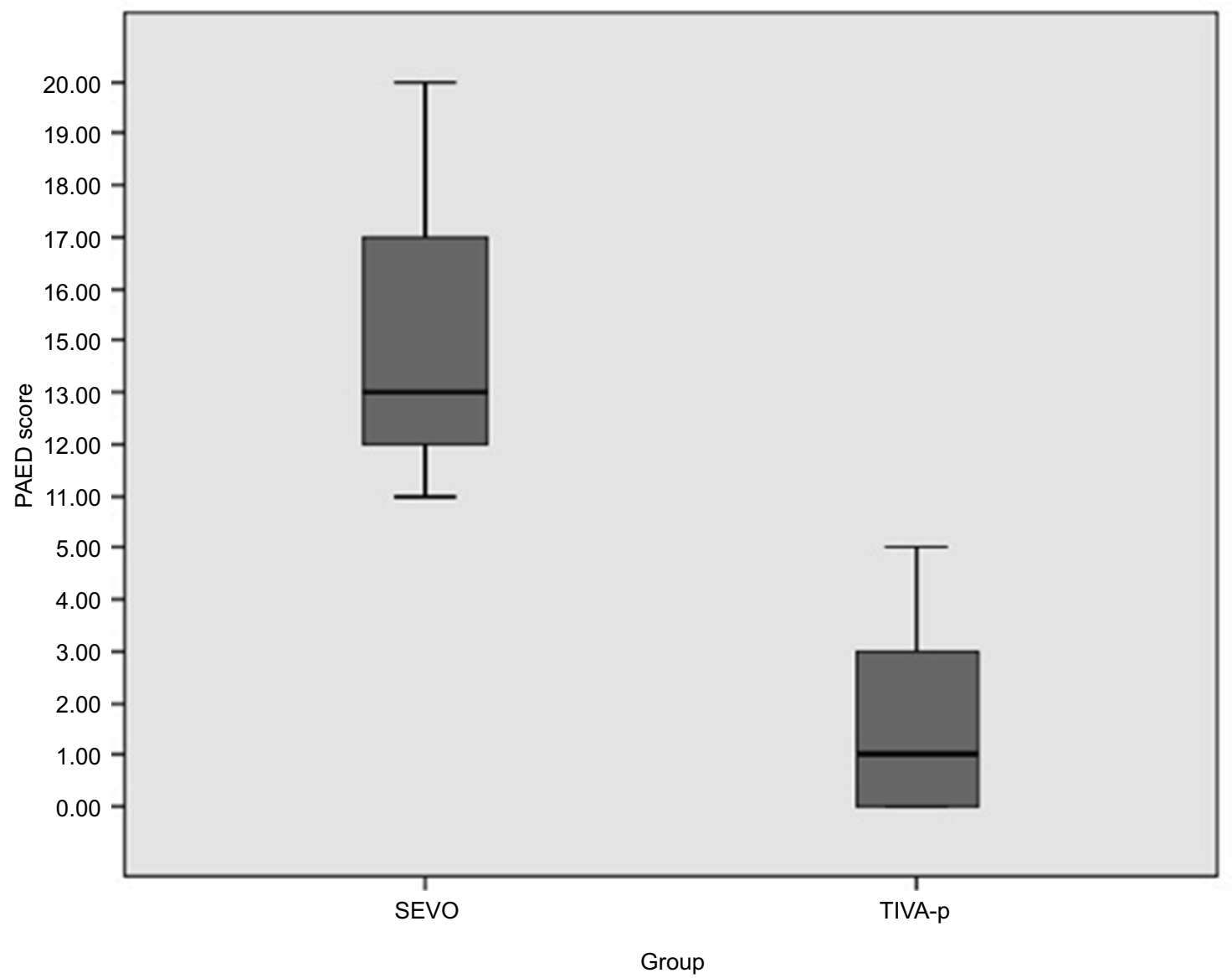

Figure 2 Comparison of the PAED scores of the groups.

Abbreviations: PAED, Pediatric Anesthesia Emergence Delirium; SEVO, sevoflurane; TIVA-p, total intravenous anesthesia with propofol.

Table 2 Comparison of recovery outcomes and parental satisfaction levels of the groups (mean \pm SD)

\begin{tabular}{llll}
\hline Postoperative outcomes & SEVO & TIVA-p & P-value \\
\hline Extubation time $^{\mathrm{a}}$ & $2(\mathrm{I}-5)$ & $2(\mathrm{I}-5)$ & 0.77 \\
FLACC score $^{\mathrm{a}}$ & $3(0-8)$ & $\mathrm{I}(0-9)$ & $0.000^{*}$ \\
PAED $\geq 10^{\mathrm{b}}$ score $^{\text {Recovery time }}$ & $38(65.5)$ & $2(3.4)$ & $0.000^{\mathrm{a}}$ \\
Parental satisfaction level $^{\mathrm{a}}$ & $13(10-20)$ & $13(9-20)$ & 0.99 \\
\hline
\end{tabular}

Notes: a Median (interquartile range). ${ }^{b} n(\%)$. *Statistically significant at $P>0.05$ value. Abbreviations: FLACC, Face, Legs, Activity, Cry, Consolability; PAED, Pediatric Anesthesia Emergence Delirium; SEVO, sevoflurane; TIVA-p, total intravenous anesthesia with propofol.

in the clinical evaluation of EA. The FLACC scale is used for pain assessment in individuals who cannot self-report their pain. ${ }^{10}$ Recent studies have frequently used the PAED scale to evaluate ED and the FLACC scale to evaluate postoperative pain after outpatient surgery under general anesthesia. ${ }^{8,13}$

The reasons for the higher incidence of ED after SEVO anesthesia are not completely clear. Multiple hypotheses associated with the phenomena of epileptiform EEG changes, temporary neurological dysfunction, rapid recovery, and responses to pain have been proposed. ${ }^{14-16}$

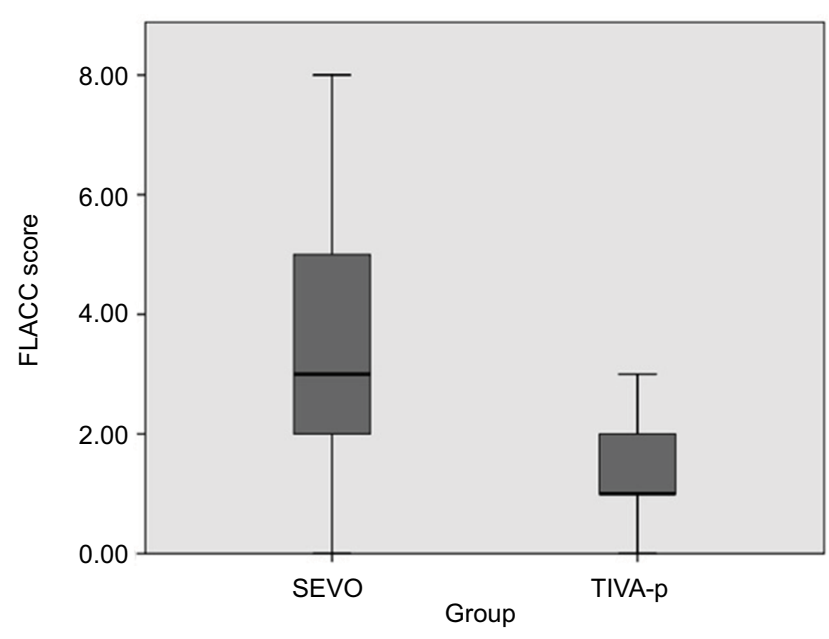

Figure 3 Comparison of the FLACC scores of the groups.

Abbreviations: FLACC, Face, Legs, Activity, Cry, Consolability; SEVO, sevoflurane; TIVA-p, total intravenous anesthesia with propofol.

The use of SEVO may be associated with cortical epileptiform EEG findings, usually without clinical symptoms. ${ }^{17}$ Persistent neurological or EEG sequelae have not been identified, and the potential morbidity of this epileptogenic 
effect is unknown. ED after SEVO anesthesia may be associated with epileptogenic activity. ${ }^{15,18}$ Yasui et al ${ }^{19}$ detected an increase in noradrenaline release in the locus coeruleus of preoptic rat brains, which has been suggested to also lead to disorientation characterized by agitation in the early stages of recovery from anesthesia. ${ }^{19}$

Another hypothesis is that rapid, early recovery may be associated with the higher incidence of ED after SEVO anesthesia. ${ }^{14}$ Psychological immaturity of preschool-aged patients has also been suggested as a cause of ED. ${ }^{20}$ Uezono et $\mathrm{al}^{14}$ reported that SEVO resulted in a shorter PACU stay than propofol. Furthermore, they suggested that rapid, early recovery after SEVO may have contributed to the incidence of agitation. In contrast, Cohen et $\mathrm{al}^{21}$ showed that the times to extubation and recovery were similar between SEVO and propofol groups, but that ED was significantly more common in the SEVO group. Consistent with their study, we did not detect differences between SEVO anesthesia and TIVA-p in terms of extubation time and recovery time. Picard et $\mathrm{al}^{6}$ reported that rapid recovery from SEVO anesthesia was not a contributing factor to ED. Our study supports this finding because a significant difference was found between the incidences of ED, but no differences were observed in extubation time and recovery time between the groups.

Postoperative pain measured by the FLACC score was affected by the type of anesthetic in this trial. We found higher FLACC scores in the SEVO group than in the TIVA-p group. Many studies support that propofol-based anesthesia reduces postoperative pain and the need for rescue analgesia. ${ }^{8,13,22}$ Chandler et $\mathrm{al}^{8}$ reported that the use of TIVA-p reduced postoperative pain measured by FLACC scores in children aged between 2 and 6 years who underwent strabismus surgery. Pieters et $\mathrm{al}^{22}$ did not find a significant difference in the incidence of ED in children randomized to anesthetic maintenance with either propofol or SEVO during adenotonsillectomy, as measured by the PAED scale; however, they found that patients in the propofol group required less pain medication in the PACU. König et $\mathrm{al}^{13}$ found no significant difference in PAED scores between groups of children aged between 2 and 12 years receiving propofol- or SEVO-based anesthetic techniques for dental procedures, but they noted a significantly greater number of PACU nursing interventions in the SEVO group, mostly for pain medication administration. However, they found a significant positive correlation between FLACC and PAED scores. Similar to the study by Chandler et al, we found a positive significant correlation between FLACC and PAED scores, with lower incidences of ED and postoperative pain in the TIVA-p group.
Rapid awakening has been suggested as one of the causes of EA. ${ }^{20,23}$ Recent studies have shown no statistically significant differences between SEVO anesthesia and propofol anesthesia in terms of extubation time and recovery time..$^{6,14,21}$ The time to extubation and the recovery time were comparable in the TIVA-p and SEVO groups in this study, which is consistent with previous reports. Kanaya et a ${ }^{24}$ indicated that extubation time was not a primary endpoint of most studies included in their meta-analysis and extubation criteria were not clearly defined in most studies. The reported differences in extubation time between propofol and SEVO groups were small and can be considered clinically insignificant. Therefore, our results and these previous findings challenge the relationship between rapid awakening and EA.

A higher ED frequency and a lower parental satisfaction were observed after SEVO-based anesthesia. We found that lower parental satisfaction could be due to children's behavior, such as presenting a bad mood and intermittent crying. Our results are consistent with those in a study conducted by Uezono et al. ${ }^{14}$ Similar to their study, parental satisfaction levels in the TIVA-p group were higher than those in the SEVO group in this study.

The main limitation of the current study is the wide age range of the patients, ranging from 3 to 7 years, which could explain the large differences in ED and FLACC scores.

\section{Conclusion}

This randomized clinical trial revealed that induction and maintenance of anesthesia with TIVA-p decreased the incidence of ED compared with SEVO anesthesia in preschoolaged children who underwent full mouth dental rehabilitation. The recovery time and time to extubation were similar for both anesthetic techniques, suggesting that rapid awakening did not fully explain the origin of ED. However, postoperative pain measured by FLACC scores could be related to the development of ED. The higher parental satisfaction with TIVA-p could be primarily due to the calm behavior of the children.

\section{Disclosure}

The authors report no conflicts of interest in this work.

\section{References}

1. Vlajkovic GP, Sindjelic RP. Emergence delirium in children: many questions, few answers. Anesth Analg. 2007;104(1):84-91.

2. Sikich N, Lerman J. Development and psychometric evaluation of the pediatric anesthesia emergence delirium scale. Anesthesiology. 2004;100(5):1138-1145.

3. Chung F. Recovery pattern and home-readiness after ambulatory surgery. Anesth Analg. 1995;80(5):896-902. 
4. Eger EI 2nd. New inhaled anesthetics. Anesthesiology. 1994;80(4): 906-922.

5. Lerman J, Sikich N, Kleinman S, Yentis S. The pharmacology of sevoflurane in infants and children. Anesthesiology. 1994;80(4): 814-824.

6. Picard V, Dumont L, Pellegrini M. Quality of recovery in children: sevoflurane versus propofol. Acta Anaesthesiol Scand. 2000;44(3):307-310.

7. Smith I, White PF, Nathanson M, Gouldson R. Propofol. An update on its clinical use. Anesthesiology. 1994;81(4):1005-1043.

8. Chandler JR, Myers D, Mehta D, et al. Emergence delirium in children: a randomized trial to compare total intravenous anesthesia with propofol and remifentanil to inhalational sevoflurane anesthesia. Paediatr Anaesth. 2013;23(4):309-315.

9. Knapp R, Gilchrist F, Rodd HD, Marshman Z. Change in children's oral health-related quality of life following dental treatment under general anaesthesia for the management of dental caries: a systematic review. Int J Paediatr Dent. 2017;27(4):302-312.

10. Malviya S, Voepel-Lewis T, Burke C, Merkel S, Tait AR. The revised FLACC observational pain tool: improved reliability and validity for pain assessment in children with cognitive impairment. Paediatr Anaesth. 2006;16(3):258-265.

11. Cravero J, Surgenor S, Whalen K. Emergence agitation in paediatric patients after sevoflurane anaesthesia and no surgery: a comparison with halothane. Paediatr Anaesth. 2000;10(4):419-424.

12. Nakayama S, Furukawa H, Yanai H. Propofol reduces the incidence of emergence agitation in preschool-aged children as well as in school-aged children: a comparison with sevoflurane. J Anesth. 2007;21(1):19-23.

13. König MW, Varughese AM, Brennen KA, et al. Quality of recovery from two types of general anesthesia for ambulatory dental surgery in children: a double-blind, randomized trial. Paediatr Anaesth. 2009;19(8):748-755.
14. Uezono S, Goto T, Terui K, et al. Emergence agitation after sevoflurane versus propofol in pediatric patients. Anesth Analg. 2000;91(3):563-566.

15. Veyckemans F. Excitation and delirium during sevoflurane anesthesia in pediatric patients. Minerva Anestesiol. 2002;68(5):402-405.

16. Woodforth IJ, Hicks RG, Crawford MR, Stephen JPH, Burke DJ. Electroencephalographic evidence of seizure activity under deep sevoflurane anesthesia in a nonepileptic patient. Anesthesiology. 1997;87(6): $1579-1582$.

17. Constant I, Seeman R, Murat I. Sevoflurane and epileptiform EEG changes. Paediatr Anaesth. 2005;15(4):266-274.

18. Messieha Z. Prevention of sevoflurane delirium and agitation with propofol. Anesth Prog. 2013;60(2):67-71.

19. Yasui Y, Masaki E, Kato F. Sevoflurane directly excites locus coeruleus neurons of rats. Anesthesiology. 2007;107(6):992-1002.

20. Aono J, Ueda W, Mamiya K, Takimoto E, Manabe M. Greater incidence of delirium during recovery from sevoflurane anesthesia in preschool boys. Anesthesiology. 1997;87(6):1298-1300.

21. Cohen IT, Finkel JC, Hannallah RS, Hummer KA, Patel KM. Rapid emergence does not explain agitation following sevoflurane anaesthesia in infants and children: a comparison with propofol. Paediatr Anaesth. 2003;13(1):63-67.

22. Pieters BJ, Penn E, Nicklaus P, Bruegger D, Mehta B, Weatherly R. Emergence delirium and postoperative pain in children undergoing adenotonsillectomy: a comparison of propofol vs sevoflurane anesthesia. Paediatr Anaesth. 2010;20(10):944-950.

23. Voepel-Lewis T, Malviya S, Tait AR. A prospective cohort study of emergence agitation in the pediatric postanesthesia care unit. Anesth Analg. 2003;96(6):1625-30, table of contents.

24. Kanaya A, Kuratani N, Satoh D, Kurosawa S. Lower incidence of emergence agitation in children after propofol anesthesia compared with sevoflurane: a meta-analysis of randomized controlled trials. JAnesth. 2014;28(1):4-11.
Journal of Pain Research

\section{Publish your work in this journal}

The Journal of Pain Research is an international, peer reviewed, open access, online journal that welcomes laboratory and clinical findings in the fields of pain research and the prevention and management of pain. Original research, reviews, symposium reports, hypothesis formation and commentaries are all considered for publication.

\section{Dovepress}

The manuscript management system is completely online and includes a very quick and fair peer-review system, which is all easy to use. Visit http://www.dovepress.com/testimonials.php to read real quotes from published authors. 